\title{
Weak Te,Te Interactions through the Looking Glass of NMR Spin-Spin Coupling**
}

\author{
Michael Bühl,* Fergus R. Knight, Anezka Kř́stková, Irina Malkin Ondík, Olga L. Malkina, \\ Rebecca A. M. Randall, Alexandra M. Z. Slawin, and J. Derek Woollins
}

Most of the tools for quantifying the extent of chemical bonding between two atoms are quantum-chemical in nature. None of them are unambiguous, however, and different analyses can lead to conflicting interpretations, even concerning the most fundamental question of whether or not atoms are linked by a chemical bond. ${ }^{[1,2]}$ One of the indicators that can be probed experimentally is the indirect spin-spin coupling constant (SSCC). For instance, observation of spinspin coupling across hydrogen bonds ${ }^{[3]}$ has been taken as evidence for covalent contributions to this kind of bonding. For atoms within the same molecule that are close in space, but not linked through a direct formal bond, the question arises, how much of the observed coupling is transmitted through a succession of bonds that eventually links them ("through-bond coupling"), and how much is due to interaction through the overlap of lone pairs ("through-space coupling"). Again, a variety of quantum-chemical tools have been developed to address this question.

A rigid scaffold that is used to achieve such spatial proximity is the naphthalene framework, in which substituents in the peri $(1,8)$ positions have a typical separation of around $3 \AA{ }^{[4]}$ Through-space $J\left({ }^{19} \mathrm{~F},{ }^{19} \mathrm{~F}\right)$ SSCCs, long known for their distance dependence,${ }^{[5]}$ have been studied in some detail in peri-difluoronaphthalenes. ${ }^{[\mathrm{b}, \mathrm{c}]}$ Similarly, $J\left({ }^{31} \mathrm{P},{ }^{31} \mathrm{P}\right)$ values in peri-bis(phosphino)naphthalenes have been attributed to through-space coupling, ${ }^{[6]}$ and $J\left({ }^{77} \mathrm{Se},{ }^{77} \mathrm{Se}\right)$ values in peri-bis(seleno) derivatives have been analyzed in detail through quantum-chemical computations. ${ }^{[7]}$ In systematic

[*] Prof. M. Bühl, Dr. F. R. Knight, R. A. M. Randall,

Prof. A. M. Z. Slawin, Prof. J. D. Woollins

EaStCHEM School of Chemistry, University of St. Andrews

North Haugh, St. Andrews, Fife KY16 9ST (UK)

E-mail: buehl@st-andrews.ac.uk

A. Krístková, Dr. O. L. Malkina

Slovak Academy of Sciences, Institute of Inorganic Chemistry 84536 Bratislava (Slovakia)

Dr. I. Malkin Ondík

Faculty of Mathematics, Physics and Informatics

Comenius University, Bratislava

84248 Bratislava (Slovakia)

[**] This work was supported by the School of Chemistry and by EaStChem via the EaStChem Research Computing facility maintained by Dr. H. Früchtl, and by Slovak grant agencies VEGA (grant 2/0079/09) and APVV (grant LPP-0326-09). We thank Prof. Bernd Wrackmeyer for suggesting the ${ }^{123} \mathrm{Te}$ NMR experiments and Dr. Vladimir G. Malkin for helpful discussions.

$\square$ Sup Supporting information for this article (including full computational and experimental details and additional graphical material) is available on the WWW under http://dx.doi.org/10.1002/anie. 201205998 studies of naphthalene (N) and acenaphthene (A) derivatives with pnictogen and chalcogen atoms in the peri positions, it became apparent that for the heavier congeners, steric repulsion is partly counterbalanced by attractive interactions. In particular with Te substituents, formally nonbonded, "across-the-bay" distances are significantly shorter than the sum of the van-der-Waals radii, which has been traced back to weak donor-acceptor interactions and the onset of 3-center-4electron (3c4e) bonding. ${ }^{[8]}$

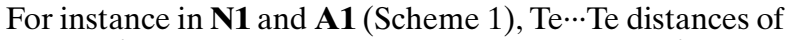
around $3.3 \AA$ are observed in the solid state (ca. $0.7 \AA$ below the sum of the vdW radii), and Wiberg bond indices ${ }^{[9]}$ (WBIs,

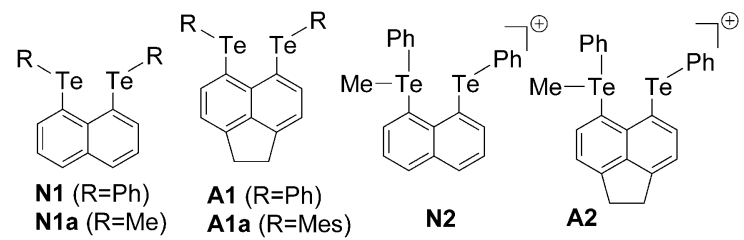

Scheme 1. Peri-napthalene ditellurides with formally nonbonded Te atoms.

a measure for the covalent character of a bond, approaching $\mathrm{WBI}=1$ for a true single bond) of around 0.15 have been computed. Slightly larger WBIs of approximately 0.18 have been obtained for cationic methylated species N2 and A2, despite a slightly longer Te-Te separation (ca. 3.4 ̊).$^{[10]}$ These unsymmetrical systems show remarkably large $J\left({ }^{125} \mathrm{Te},{ }^{125} \mathrm{Te}\right)$ SSCCs, formally ${ }^{4} J$ values, of $1093.0 \mathrm{~Hz}$ and $945.8 \mathrm{~Hz}$ in $\mathbf{N 2}$ and $\mathbf{A 2}$, respectively. ${ }^{[10]}$ We now report even larger couplings in $\mathbf{N 1}$ and $\mathbf{A 1}$, along with computational conformational analysis underlining the potential of this property as a structural and interpretative method.

The computed (ZORA-SO/BP//B3LYP level $)^{[11]}$ $J\left({ }^{125} \mathrm{Te},{ }^{125} \mathrm{Te}\right)$ SSCCs in $\mathbf{N 2}$ and $\mathbf{A 2}(1490$ and $1377 \mathrm{~Hz}$, respectively) are noticeably overestimated with respect to the experiment, but are in the right order of magnitude and reproduce the relative tendency rather well. At the same level, $J$ values of 2779 and $1543 \mathrm{~Hz}$ were predicted for $\mathbf{N 1}$ and A1, respectively, showing a larger discrimination between the two types of compounds. As will be shown below, this discrimination is largely due to subtle conformational effects.

Because the two Te sites in $\mathbf{N 1}$ and $\mathbf{A 1}$ are magnetically equivalent in solution, direct observation of $J\left({ }^{125} \mathrm{Te},{ }^{125} \mathrm{Te}\right)$ is not possible, but the coupling can be detected as satellites in the ${ }^{123} \mathrm{Te}$ NMR spectrum (see experimental details). With this technique, $J\left({ }^{123} \mathrm{Te},{ }^{125} \mathrm{Te}\right)$ values of 2077 and $1750 \mathrm{~Hz}$ are 
obtained for $\mathbf{N 1}$ and $\mathbf{A 1}$, respectively. Neglecting primary and secondary isotope effects, these values correspond to 2505 and $2110 \mathrm{~Hz}$, respectively, in $J\left({ }^{125} \mathrm{Te},{ }^{125} \mathrm{Te}\right)$. While the computed value for $\mathbf{N 1}$ is again overestimated with respect to the experiment, that for $\mathbf{A 1}$ is underestimated, but overall, the predicted tendency is confirmed qualitatively.

To gain deeper insights into the factors that govern the magnitude of these $J$ values, additional computations were performed (Table 1). Typical for NMR properties of heavier elements, ${ }^{[12]}$ the theoretical $J$ couplings are quite sensitive to

Table 1: Computed $J\left({ }^{125} \mathrm{Te},{ }^{125} \mathrm{Te}\right)$ couplings in peri-naphthalene ditelluride derivatives.

\begin{tabular}{|c|c|c|c|c|c|}
\hline Entry & Compound & Level of theory ${ }^{[a]}$ & $J_{F C}^{[b]}[H z]$ & $\int_{\mathrm{PSO}}{ }^{[\mathrm{c}]}[\mathrm{Hz}]$ & $\int_{\text {tot }}^{[d]}[\mathrm{Hz}]$ \\
\hline 1 & N1 & NR/BP//B3LYP & 1872 & -2 & 1870 \\
\hline 2 & N1 & ZORA/BP//B3LYP & 2874 & -11 & 2863 \\
\hline 3 & N1 & ZSO/BP//B3LYP & 2858 & -79 & 2779 \\
\hline 4 & N1 & ZSO/BP//PBEO & 3373 & -38 & 3335 \\
\hline 5 & N1 & ZSO/PBEO//PBEO & 3707 & -46 & 3661 \\
\hline 6 & N1 a & ZSO/BP//B3LYP & 2739 & -48 & 2691 \\
\hline 7 & A1 & ZSO/BP//B3LYP & 2640 & -36 & 2604 \\
\hline 8 & $A 1^{[\mathrm{e}]}$ & ZSO/BP//B3LYP & 1713 & -170 & 1543 \\
\hline
\end{tabular}

[a] Notation "level of NMR computation//level of geometry optimization" (CCt conformer except where otherwise noted); NR: nonrelativistic, ZSO: Zora-spin-orbit, TZ2P basis employed throughout. [b] Sum of Fermi-contact and spin-dipolar part. [c] Paramagnetic spin-orbit part. [d] Total J coupling. [e] AB conformer.

relativity (compare entries $1-3$ in Table 1 ), level of geometry optimization (compare entries 3 and 4), exchange-correlation functional (compare entries 4 and 5), and rather strongly on the conformation (compare entries 7 and 8).

The conformations of each phenyl group with respect to the naphthalene plane can be classified as roughly perpendicular (A), in-plane (B), or in-between (C), and both can be cis (c) or trans (t) to each other. ${ }^{[8]}$ In the solid, N1 and A1 adopt $\mathrm{CCt}$ and $\mathrm{AB}$ conformations, respectively (see Figure S1 in the Supporting Information). For the smaller methyl derivative N1a, for which a $J$ value very similar to that of $\mathbf{N 1}$ is computed (compare entries 3 and 6 in Table 1), a detailed conformational analysis was carried out. Results as a function of the two $\mathrm{C}^{9}-\mathrm{C}-\mathrm{Te}-\mathrm{C}(\mathrm{Me})$ dihedral angles are summarized in two-dimensional (2D) Ramachandran-type plots (Figure 1).

The first of the 2D plots represents the potential energy, with the minimum labeled as CCt. There is a huge flat area within just $1 \mathrm{kcal} \mathrm{mol}^{-1}$ (light yellow) which extends well into what would be classified as $\mathrm{AB}$ conformation, consistent with the structural variety observed in the solid for different members of this family. ${ }^{[8]}$ The second of the $2 \mathrm{D}$ plots represents the $J$ coupling surface, which looks quite different. Here, a structure with CCt conformation would have a $J$ value of around $2500 \mathrm{~Hz}$, similar to what is found for $\mathbf{N 1}$; a structure in the $\mathrm{AB}$ region would be much lower (ca. $1500 \mathrm{~Hz}$ ). This situation is reminiscent of that in the Se congeners, although the $J\left({ }^{77} \mathrm{Se},{ }^{77} \mathrm{Se}\right)$ values computed for the corresponding stationary points are much smaller. ${ }^{[7,13]}$

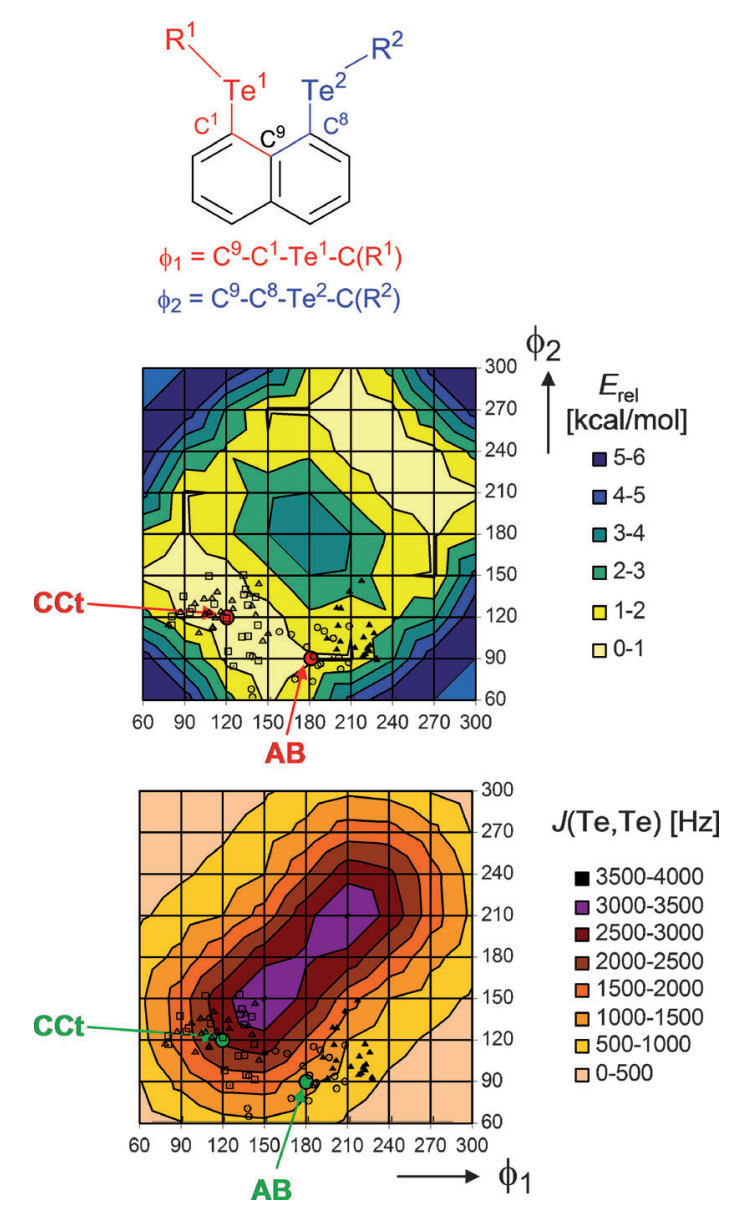

Figure 1. Relative energies (middle, B3LYP level) and $\left({ }^{125} \mathrm{Te},{ }^{125} \mathrm{Te}\right.$ ) coupling constants (bottom, ZORA-SO/BP level) in $\mathrm{N} 1$ a as a function of the conformation, as defined by the two dihedral angles specified at the top $\left(R^{1}=R^{2}=M e\right.$, all other parameters B3LYP-optimized). Open and filled black symbols: conformations visited during a molecular dynamic simulation (see main text).

From the flatness of the potential energy surface, it can be anticipated that the systems will sample large areas in the conformational space around the minima. This expectation is borne out in a short molecular dynamics (MD) simulation of N1 a at the B3LYP level, in the course of which a large territory on the $2 \mathrm{D}$ map is visited. The black symbols in Figure 1 are snapshots taken every 100 fs between $2-10$ ps of total MD simulation starting from the $\mathrm{CCt}$ minimum, demonstrating that not only the vicinity of this minimum is sampled, but also slightly higher-lying areas around an $\mathrm{AB}$ conformation. The mean $J$ coupling computed over all these snapshots is reduced significantly, by around $900 \mathrm{~Hz}$, from the equilibrium value in Table 1 (ca. $2700 \mathrm{~Hz}$ ). While this thermal correction would need much longer simulation times for full convergence (see Figure S2), it is clear that single, static NMR computations will not be sufficient to reach a quantitative agreement with the experiment. ${ }^{[14]}$ Taken together, however, the computational data enable a consistent interpretation of the observations: In solution, $\mathbf{N 1}$ and $\mathbf{A 1}$ are rather fluxional, and the observed variation in the $J\left({ }^{125} \mathrm{Te},{ }^{125} \mathrm{Te}\right)$ SSCCs are indicated to arise from subtle shifts in the populations of few conformers (or rather, larger areas in conformational phase 
space around them). Because the $J$ coupling changes so dramatically with the conformations (bottom of Figure 1), it is a sensitive probe into structure and dynamics of this kind of system.

A mean $J\left({ }^{125} \mathrm{Te},{ }^{125} \mathrm{Te}\right)$ value of more than $2500 \mathrm{~Hz}$, as observed for $\mathbf{N 1}$, is quite large for a ${ }^{4} J$ coupling between formally nonbonded Te atoms. The largest SSCC between bonded $\mathrm{Te}$ atoms is, to our knowledge, ${ }^{1} J\left({ }^{125} \mathrm{Te},{ }^{125} \mathrm{Te}\right)=$ $4395 \mathrm{~Hz}$ in $\mathrm{Te}_{2}{ }^{2-} \cdot{ }^{[15]}$ Similar values can be reached in N1a (Figure 1) and are even exceeded in one of the instantaneous MD snapshots. Why are these formal ${ }^{4} J$ couplings so large? When the naphthalene moiety in the B3LYP geometry of N1 is deleted and the Te atoms capped with $\mathrm{H}$ atoms, the computed $J$ value even increases from $2779 \mathrm{~Hz}$ to $3101 \mathrm{~Hz}$. Transmission through the intermittent TeC- and CC-bonds thus plays only a minor role.

The same conclusion can be drawn from analysis of the Te-Te coupling pathway in N1a through inspection of the coupling deformation density (CDD): ${ }^{[16]}$ Reminiscent of the situation in ${ }^{n} \mathrm{~J}(\mathrm{P}, \mathrm{P})$ couplings $(n=2,3),{ }^{[16]}$ the overlap of the lone pairs (lps) is the determining mechanism, rather than transmission through the organic framework (Figure 2). The

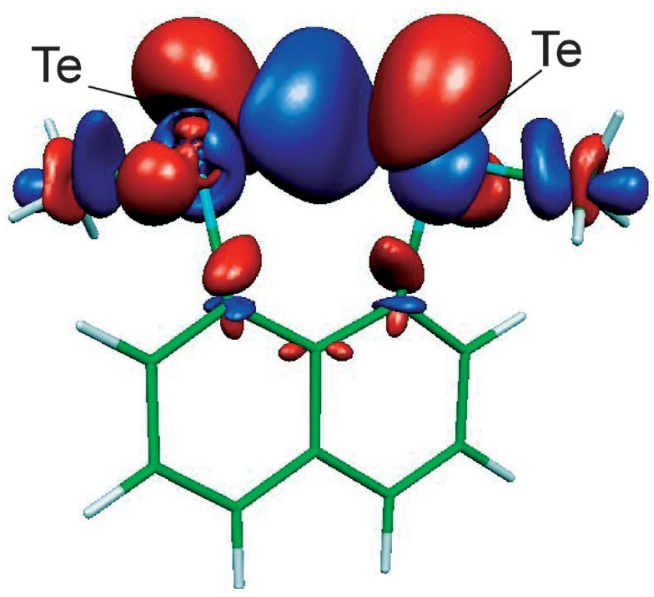

Figure 2. Total coupling deformation density (CDD) in $\mathbf{N 1 a}$. (DKH-1/ $\mathrm{BP} / \mathrm{Hirao} / \mathrm{II}$ level, isosurfaces plotted for $\mathrm{CDD}=6.0$ ).

CDD topology was obtained for the first time at a relativistic level (the first order Douglas-Kroll-Hess, DKH-1). Because it is very similar to its nonrelativistic counterpart (see Figure S3 in the Supporting Information), the analysis of $J\left({ }^{125} \mathrm{Te},{ }^{125} \mathrm{Te}\right)$ performed at the nonrelativistic level should give a qualitatively correct picture. At the nonrelativistic BP level, the total calculated FC part of $J\left({ }^{125} \mathrm{Te},{ }^{125} \mathrm{Te}\right)$ is $2038 \mathrm{~Hz}$, the largest part of which (in a localized MO formalism with Pipek-Mezey localization $^{[17]}$ ) stems from the two "sp-like" ${ }^{[18]}$ Te lone pairs $(+2359 \mathrm{~Hz}$ in total), with smaller contributions from the four $\mathrm{Te}-\mathrm{C}$ bonds $(-301 \mathrm{~Hz})$ and negligible ones from the two " $\mathrm{p}$ type" Te lone pairs $(21 \mathrm{~Hz}$, see Figure S4 in the Supporting Information).

It is noteworthy that the Te,Te coupling path in the CDD analysis extends noticeably into the "dead ends" of the $\mathrm{Te}^{-}$ $\mathrm{C}(\mathrm{Me})$ bonds, rather than the conjugated aromatic moiety.
This may be taken as evidence for the importance of the $\operatorname{lp}(\mathrm{Te}) \rightarrow \sigma^{*}(\mathrm{TeC})$ interaction that is responsible for the onset of $3 \mathrm{c} 4 \mathrm{e}$ bonding and the noticeable WBI ${ }^{[8,19]}$ In this context, it is interesting to note that at the same nonrelativistic level, a bond-critical point is located between the two Te atoms in N1 a in an atoms-in-molecules analysis. ${ }^{[20]}$

The variation in the computed $J$ values can be rationalized through the extent of overlap between the densities of the Te lone pairs (Figure S5). Compared to typical "through-space" ${ }^{n} J\left({ }^{31} \mathrm{P},{ }^{31} \mathrm{P}\right)$ couplings, the Te,Te couplings of this study are larger by at least one order of magnitude. ${ }^{[13]}$ From computed valence s-orbital densities (see the Supporting Information) and the gyromagnetic ratios, one would expect $\mathrm{Te}, \mathrm{Te}$ couplings to be approximately seven times larger than the corresponding $J(\mathrm{P}, \mathrm{P})$. Further enhancement is likely due to the increased polarizability and the onset of multicenter bonding in the Te systems.

In the course of our studies, we noticed that the mesityl derivative A1 a showed an even larger Te, Te coupling than N1 or A1, namely $J\left({ }^{125} \mathrm{Te},{ }^{125} \mathrm{Te}\right)=3398 \mathrm{~Hz}$. This value led us to suspect that, unlike A1 with its $\mathrm{AB}$ conformation, ${ }^{[8]} \mathbf{A 1} \mathbf{a}$ would rather adopt a CCt conformation, an expectation that was borne out in a subsequent structure determination by $\mathrm{X}$ ray crystallography (Figure 3). ${ }^{[21]}$ These findings underscore the potential of Te,Te-coupling constants as an analytical tool.

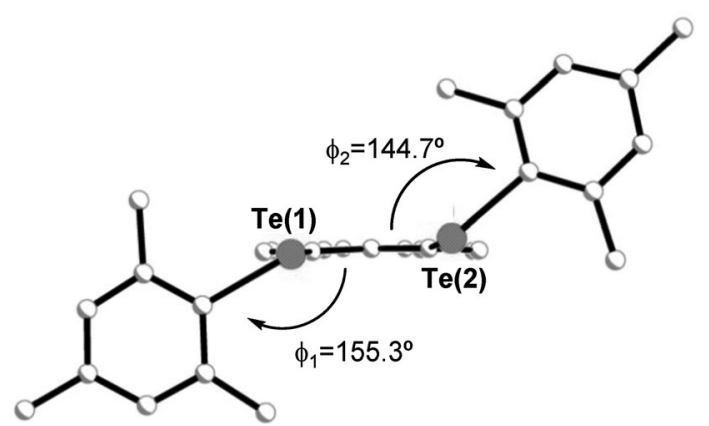

Figure 3. The crystal structure of 5,6-bis (mesityltelluro)acenaphthene (A1 a, $\mathrm{H}$ atoms omitted for clarity), including the key dihedral angles for classification as CCt conformation. See the Supporting Information for further details.

In summary, a combination of NMR and DFT techniques has been used to study the interactions between formally nonbonded, but spatially close Te atoms. Weak donoracceptor interactions in the peri-naphthalene system, which mark the onset of $3 \mathrm{c} 4 \mathrm{e}$ bonding, reinforce the Te,Te couplings and lead to unusually large $J\left({ }^{125} \mathrm{Te},{ }^{125} \mathrm{Te}\right)$ values. This property turns out to be a sensitive probe ("looking glass"), not only into the electronic structure underlying the bonding situation, but also into the particular conformations that ensue. In the broader context of well-known through-space spin-spin coupling, this conformational aspect is a new facet worth exploring. Studies are under way to probe how substituents at the phenyl rings can modulate the bonding and the concomitant NMR properties, ever broadening our knowledge of the very foundation of chemistry, the chemical bond. 


\section{Experimental Section}

Geometry optimizations and Born-Oppenheimer MD simulations were carried out at the B3LYP/6-31G* level (augmented SDD on Te), $J$ values computed ${ }^{[22]}$ at the ZORA-Spinorbit/BP86/TZ2P level (which has performed well for the computation of SSCCs involving fourth-row and heavier elements) ${ }^{[23]}$ or at the nonrelativistic BP86 level (TZVP on Te, IGLO-II on C,H) ${ }^{[1]]}{ }^{123}$ Te NMR spectra were recorded on a Jeol GSX $270 \mathrm{MHz}$ spectrometer with $\delta(\mathrm{Te})$ referenced to external diphenyl ditelluride. ${ }^{123} \mathrm{Te}$ NMR $\left(70.7 \mathrm{MHz}, 25^{\circ} \mathrm{C}\right.$, PhTe$\mathrm{TePh}): \mathbf{N 1}\left(\mathrm{CDCl}_{3}\right): \delta=620.3 \mathrm{ppm}\left(\mathrm{s},{ }^{4} J\left({ }^{123} \mathrm{Te},{ }^{125} \mathrm{Te}\right)=2077.4 \mathrm{~Hz}\right) . \mathbf{A 1}$ $\left(\mathrm{CDCl}_{3}\right): \delta=586.6 \mathrm{ppm}\left(\mathrm{s},{ }^{4} \mathrm{~J}\left({ }^{123} \mathrm{Te},{ }^{125} \mathrm{Te}\right)=1750.4 \mathrm{~Hz}\right.$; see Figure $\mathrm{S} 8$ in the Supporting Information for the spectra), $\mathbf{A 1 a}\left(\mathrm{CDCl}_{3}\right): \delta=$ $362.9 \mathrm{ppm}\left(\mathrm{s}, J\left({ }^{123} \mathrm{Te}-{ }^{125} \mathrm{Te}\right) 2818.5 \mathrm{~Hz}\right.$; see Supporting Information for experimental details).

Received: July 26, 2012

Revised: November 14, 2012

Published online: January 23, 2013

Keywords: naphthalenes - quantum-chemical computation . tellurium · through-bond interactions . through-space interactions

[1] P. Ball, Nature 2011, 469, 26.

[2] For example: a) S. Alvarez, R. Hoffmann, C. Mealli, Chem. Eur. J. 2009, 15, 8358; b) H. S. Rzepa, Nat. Chem. 2009, 1, 510.

[3] A. D. Dingley, S. Grzesiek, J. Am. Chem. Soc. 1998, 120, $8293-$ 8297.

[4] a) P. Kilian, F. R. Knight, J. D. Woollins, Chem. Eur. J. 2011, 17, 2302; b) P. Kilian, F. R. Knight, J. D. Woollins, Coord. Chem. Rev. 2011, 255, 1387.

[5] For example: a) L. Ernst, K. Ibdrom, Angew. Chem. 1995, 107, 2010-2012; Angew. Chem. Int. Ed. Engl. 1995, 34, 1881-1882, and references therein; b) F. D. Mallory, C. W. Mallory, K. E. Butler, M. B. Lewis, A. Q. Xia, E. D. Luzik, Jr., L. E. Fredenburgh, M. N. Ramanjulu, Q. N. Van, M. N. Francl, D. A. Freed, C. C. Wray, C. Hann, M. Nerz-Stormes, P. J. Caroll, L. E. Chirlian, J. Am. Chem. Soc. 2000, 122, 4108-4116; c) J. E. Peralta, V. Barone, R. H. Contreras, J. Am. Chem. Soc. 2001, 123, $9162-9163$.

[6] For example: a) S. A. Reiter, S. D. Nogai, K. Karaghiosoff, H. Schmidbaur, J. Am. Chem. Soc. 2004, 126, 15833-15843; b) P. Kilian, A. M. Z. Slawin, J. D. Woollins, Phosphorus Sulfur Silicon Relat. Elem. 2004, 179, 999.

[7] W. Nakanishi, S. Hayashi, Chem. Eur. J. 2008, 14, 5645-5655.

[8] L. K. Aschenbach, F. R. Knight, R. A. M. Randall, D. B. Cordes, A. Baggott, M. Bühl, A. M. Z. Slawin, J. D. Woollins, Dalton Trans. 2012, 41, 3141-3153.

[9] K. Wiberg, Tetrahedron 1968, 24, 1083.

[10] F. R. Knight, R. A. M. Randall, K. S. A. Arachchige, L. Wakefield, J. M. Griffin, S. E. Ashbrook, M. Bühl, A. M. Z. Slawin, J. D. Woollins, Inorg. Chem. 2012, 51, 11087-11097.

[11] See the Supporting Information for details and references.

[12] For example: J. Autschbach, S. Zheng, Annu. Rep. NMR Spectrosc. 2009, 67, 1-95.

[13] Couplings involving different sets of nuclei are best compared in terms of reduced coupling constants $K$; for $1,8-\mathrm{C}_{10} \mathrm{H}_{6} \mathrm{X}_{2}$ the following $K$ values are obtained at the ZSO/BP86/TZ2P level (in $10^{19} \mathrm{~kg} \mathrm{~m}^{-2} \mathrm{~s}^{-2} \mathrm{~A}^{-2}$, in bold $): \mathrm{X}=\mathrm{F}, \boldsymbol{K}\left({ }^{\mathbf{1 9}} \mathbf{F},{ }^{\mathbf{1 9}} \mathbf{F}\right)=\mathbf{3 . 7}(\mathrm{J}=38.9 \mathrm{~Hz})$; $\mathrm{X}=\mathrm{PMe}_{2}, \quad \boldsymbol{K}\left({ }^{\mathbf{3 1}} \mathbf{P},{ }^{31} \mathbf{P}\right)=\mathbf{1 0 7 . 8} \quad(J=213 \mathrm{~Hz}) ; \quad \mathrm{X}=\mathrm{SeMe}$, $\boldsymbol{K}\left({ }^{77} \mathrm{Se},{ }^{77} \mathrm{Se}\right)=\mathbf{7 7 6} \quad(\mathrm{CCt} \quad$ isomer, $\quad J=341 \mathrm{~Hz}) ; \quad \mathrm{X}=\mathrm{TeMe}$, $\boldsymbol{K}\left({ }^{125} \mathbf{T e},{ }^{125} \mathbf{T e}\right)=2220(\mathrm{CCt}$ isomer, $J=2691 \mathrm{~Hz})$. By moving down one row in the periodic table, $K$ increases significantly, for example, by a factor of three between the $\mathrm{Se}$ and $\mathrm{Te}$ congeners.

[14] The reasonably good accord between the ZSO/BP//B3LYP result for $\mathbf{N} 1(2779 \mathrm{~Hz}$, Table 1$)$ with the experimental result $(2505 \mathrm{~Hz})$ may thus be somewhat random.

[15] Converted from the ${ }^{1} J\left({ }^{123} \mathrm{Te},{ }^{125} \mathrm{Te}\right)$ value of $3645 \mathrm{~Hz}$ in liquid ammonia, given in: a) M. Björgvinsson, G. J. Schrobilgen, Inorg. Chem. 1991, 30, 2540-2547; note that a ${ }^{1} J\left({ }^{125} \mathrm{Te},{ }^{125} \mathrm{Te}\right)$ value of $3568 \mathrm{~Hz}$ has been reported for solid $\left(\mathrm{NH}_{4}\right)_{2} \mathrm{Te}_{2}:$ b) R. J. Batchelor, F. W. B. Einstein, I. D. Gay, C. H. W. Jones, R. D. Sharma, Inorg. Chem. 1993, 32, 4378-4383.

[16] O. L. Malkina, A. Kř́stková, E. Malkin, S. Komorovsky, V. G. Malkin, Phys. Chem. Chem. Phys. 2011, 13, 16015-16021, and references therein.

[17] J. Pipek, P. G. Mezey, J. Chem. Phys. 1989, 90, 4916; J. Pipek, Int. J. Quantum Chem. 1989, 36, 487.

[18] In an analysis of a natural bond orbital (A. E. Reed, F. L. A. Curtiss, F. Weinhold, Chem. Rev. 1988, 88, 899-926), this localized orbital has an even larger s-character of around $75 \%$.

[19] If the FC part of $J\left({ }^{125} \mathrm{Te},{ }^{125} \mathrm{Te}\right)$ in $\mathbf{N 1}$ a is subjected to an NBO analysis (J. Autschbach, J. Chem. Phys. 2008, 128, 164112) at the scalar ZORA level, the $J$ coupling is clearly dominated by the four lone pairs, with the two "sp-type" and "p-type" lone pair NLMOs contributing each around $1000 \mathrm{~Hz}$ and $400 \mathrm{~Hz}$, respectively. A similar picture is obtained for the FC part of $J\left({ }^{125} \mathrm{Te},{ }^{125} \mathrm{Te}\right.$ ) in N1a in a natural $J$ coupling analysis (S. J. Wilkens, W. M. Westler, J. L. Markley, F. Weinhold, J. Am. Chem. Soc. 2001, 123, 12026-12036) at the nonrelativistic BP level, where roughly half of the $J$ coupling is indicated to come from the Lewis structure, the other half from delocalization. The Lewis and total NLMO contributions are dominated by the "sp" lone pairs, whereas the single largest delocalization contributions stem from the "sp-type" $l p(\mathrm{Te}) \rightarrow \sigma^{*}(\mathrm{TeC})$ interactions (ca. $400 \mathrm{~Hz}$ each).

[20] The Quantum Theory of Atoms in Molecules (Eds.: C. F. Matta, R. J. Boyd), Wiley-VCH, Weinheim, 2007; for the critical point in N1 a, $\rho$ and $\nabla \rho$ values are 0.022 au and 0.033 au, respectively, that is, around $20 \%$ and $100 \%$, respectively, of the corresponding values for the $\mathrm{Te}-\mathrm{C}$ bonds; very similar values are obtained for N1.

[21] This is the only minimum that can be located for A1a at the B3LYP level (computed $J$ value of $2738 \mathrm{~Hz}$ ); apparently, the Me substituents help to sample the area of maximum $J$ in $J$ space (around $150^{\circ} / 150^{\circ}$ in Figure 1).

[22] a) J. Autschbach, T. Ziegler, J. Chem. Phys. 2000, 113, 936; b) J. Autschbach, J. Chem. Phys. 2008, 129, 094105.

[23] See for instance: a) J. Autschbach, Struct. Bonding (Berlin) 2004, 112, 1-48; b) A. Bagno, G. Casella, G. Saielli, J. Chem. Theory Comput. 2006, 2, 37-46; c) F. Chen, G. M. Bernard, R. G. Cavell, R. McDonald, M. J. Ferguson, R. E. Wasylishen, J. Am. Chem. Soc. 2010, 132, 5479-5493; d) S. Moncho, J. Autschbach, J. Chem. Theory Comput. 2010, 6, 223-234. 